\section{Microbial growth in vials of Botulinum toxin following use in clinic}

\begin{abstract}
Purpose Botulinum toxin has various applications in ophthalmology and is used in the outpatient department, where it is often exposed to room temperatures for 3-4 h. The manufacturer's recommendations are that the reconstituted toxin be stored in a refrigerator when not in use and discarded $4 \mathrm{~h}$ after reconstitution. The aim of our study was to examine such used bottles of Botulinum toxin for microbial growth after $4 \mathrm{~h}$ at room temperature.
\end{abstract}

Methods and Results Eleven consecutive bottles of Botulinum toxin used with aseptic precautions in the Ophthalmology outpatient clinics were exposed to room temperatures for $4 \mathrm{~h}$. These were subsequently analysed for microbial growth. No growth was directly obtained from any of the Botulinum toxin bottles during our study.

Conclusions This pilot study suggests that if aseptic precautions are followed during the use of Botulinum toxin, the contents of the bottle remain sterile despite being exposed to room temperatures for up to $4 \mathrm{~h}$. This has implications on the use of the reconstituted toxin after the recommended $4 \mathrm{~h}$.

Eye (2007) 21, 995-997; doi:10.1038/sj.eye.6702465; published online 2 June 2006

Keywords: Botulinum; reconstituted; microbiological assay

\section{Introduction}

Botulinum A toxin (BTX-A) (Dysport, Botox) is frequently used in most Ophthalmology outpatient departments for a variety of ocular problems. These include blepharospasm, ${ }^{1}$ hemifacial spasm, ${ }^{2}$ functional epiphora, ${ }^{3}$ facial nerve palsy with exposure keratopathy, temporary correction of entropion, ${ }^{4}$ strabismus, and lid retraction in dysthyroid eye disease.
J Menon and A Murray

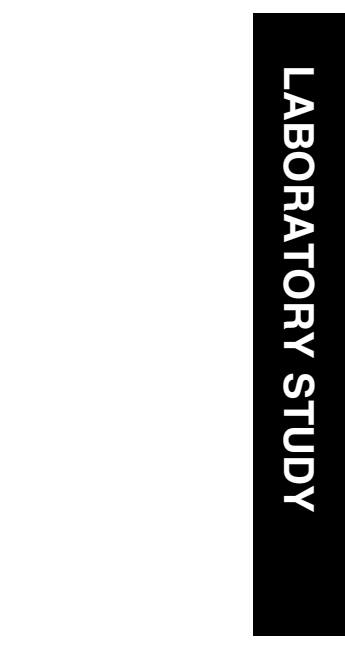

BTX-A is commercially available as two preparations: Botox (Allergan) and Dysport (Ipsen). The manufacturer's recommend that once opened the contents of the vial should be administered within $4 \mathrm{~h}$. They further recommend that the opened vial should be stored in the refrigerator when not in use during this $4 \mathrm{~h}$ period.

As is common practice in the UK, a vial of the toxin is used for more than one patient during a clinic and may often be reused during a $12 \mathrm{~h}$ period. To the best of our knowledge, a significant number of clinics do not follow the manufacturer's guidelines and the vial is allowed to remain at room temperature between use during the length of the normal clinic. The aim of our pilot study was to analyse the sterility of the contents of a used bottle of Botulinum toxin after it had been exposed to room temperature for $4 \mathrm{~h}$ (to simulate the average length of a clinic).

\section{Materials and methods}

Eleven consecutive bottles of BTX-A (Dysport) used in the outpatient department were entered into this study for microbiological analysis. All bottles were handled according to a previously agreed protocol. The rubber top of each bottle was cleaned using an alcohol wipe (sterets) before each use. For each aliquot of toxin drawn, a separate sterile needle was used to puncture the rubber top. This meant that the rubber top was punctured more than once. Most of the bottles were used two or three times in the clinic. Once opened, the bottle was kept at room temperature for $4 \mathrm{~h}$ to mirror the average length of a clinic. The bottle was then stored in a refrigerator at $3-5^{\circ} \mathrm{C}$ for $5-7$ days. The duration of refrigerated storage was agreed at 5-7 days after consulting our microbiologists to maximise the possibility of any microbiological growth in these bottles.
Department of

Ophthalmology,

Birmingham and Midland

Eye Centre, Birmingham, UK

Correspondence: J Menon, 51 Field Avenue, Northfield, Birmingham B31 1PD, UK Tel: + 4401214777557 ; Fax: + 4401215076791 E-mail: jayshreemenon@ aol.com

Received: 25 October 2005 Accepted: 21 April 2006 2006 Published online: 2 June 
The contents of the bottles were then plated by the microbiologists using standard techniques on blood agar, chocolate agar, Sabouraud agar and nutrient broth. The blood and chocolate agar, were incubated in $\mathrm{CO}_{2}$ at $37^{\circ} \mathrm{C}$ and viewed at $48 \mathrm{~h}$ and 1 week after plating. The Sabouraud agar was incubated at $30^{\circ} \mathrm{C}$ in air and again viewed at $48 \mathrm{~h}$ and 1 week. The nutrient broth was incubated for $48 \mathrm{~h}$ at $37^{\circ} \mathrm{C}$ in $\mathrm{CO}_{2}$ and subcultured if it appeared cloudy.

\section{Results}

No growth was detected on any plate or within the broth from eight bottles. In the remaining three bottles, growth was detected on one of the culture media only (ie on blood agar or chocolate agar). In each of these three cases, there was no growth directly associated with the primary inoculum. Any growth seen on the blood agar or chocolate agar plates were seen distant from the site of the primary inoculum. This implied that the growth in these cases were contaminants that occurred at the time of plating rather than from the Botulinum bottle itself. No growth therefore was directly obtained from the used bottles of Botulinum toxin after a week of incubation.

\section{Discussion}

In the UK, commercially available preparations of BTX-A contain $100 \mathrm{IU}$ (Botox) or $500 \mathrm{IU}$ (Dysport) in each vial. In most clinical applications of the toxin in ophthalmology, the dose used per patient varies between 2.5 and 50 IU (Botox) or 10 and $240 \mathrm{IU}$ (Dysport). For example, a patient with involutional entropion may require 5-10 IU (Botox) or 20-40 IU (Dysport) per eye. On the other hand, in a case with blepharospasm, the dose required may be 20-40 IU (Botox) or 80-160 IU (Dysport). The dose used in each case is largely dictated by the clinical condition itself and the individual patient's response. Thus, each such vial contains enough toxin for two or more patients. With an average cost of $£ 130-£ 165$ per vial, a significant amount of this expensive drug would be discarded as it is not completely used in the recommended $4 \mathrm{~h}$ period. Some clinicians have, therefore, often reused a single vial for multiple patients within $12 \mathrm{~h}$ of its first use (eg a morning and afternoon clinic on the same day).

This pilot study aimed to analyse the sterility of the contents within a used bottle of Botulinum toxin, if aseptic precautions were followed during each use. After exposing the bottle to $4 \mathrm{~h}$ of room temperature as is the practice in many clinics, the used bottle was stored in the refrigerator for 5-7 days before being plated on to culture media.

No direct growth was obtained from any of the 11 bottles of BTX-A in this study.
The results of this small study suggest that the contents of the used Botulinum toxin bottle remain sterile even after multiple use and exposure to room temperature provided simple aseptic precautions are followed. In our series, we used alcohol wipes (sterets) to wipe the bottle top before each use as recommended by the manufacturer's. A separate sterile needle was used for each aliquot of toxin withdrawn.

Although the number of vials studied was small, it nevertheless confirms that the contents of the toxin vials remain sterile after $4 \mathrm{~h}$ at room temperature. Despite a further storage for 5 days in the refrigerator at $3-5^{\circ} \mathrm{C}$, the reconstituted solution in the vials remained sterile. To our knowledge, there has been no published study that has analysed the possibility of microbial growth in Botulinum toxin bottles after prolonged exposure to room temperature.

The safety of reused solutions of Botulinum toxin has often been questioned particularly from the point of sterility. A further issue has also been of the acceptable length of time for reuse of the toxin. From our study, we are able to conclude that the contents in the used bottle of Botulinum toxin remain sterile after exposure to $4 \mathrm{~h}$ of room temperature and a further storage at temperatures of $3-5^{\circ} \mathrm{C}$ for at least 5 days. However, a larger study would be required to confirm this.

Other studies in animal and human models have examined the issue of the potency of the reconstituted toxin and have repeatedly confirmed that this is unaltered even after 2 weeks. Jabor et al ${ }^{5}$ concluded that the reconstituted toxin had the same initial potency and duration of action even after 2 weeks of storage. Sloop et $a l^{6}$ came to the same conclusion in a study using electromyographic studies on human volunteers. A large multicentre double-blind study by Hexsel et $\mathrm{al}^{7}$ confirmed that the potency and the efficacy are unaltered up to 6 weeks after reconstitution.

In conclusion, our pilot study confirms that the commonly followed practice in the UK of storing the reconstituted toxin of BTX-A at room temperatures for up to $4 \mathrm{~h}$ does not compromise its sterility. It also confirms that such a solution further stored in the refrigerator at $3-5^{\circ} \mathrm{C}$ remains sterile for at least 5 days after reconstitution. Previous studies in human and animal models have established that the potency of the reconstituted toxin administered after 2 weeks of storage is undiminished and compares with that of the freshly prepared toxin.

\section{References}

1 Calace P, Cortese G, Piscoppo R, Della Volpe G, Gagliardi V, Magli A et al. Treatment of blepharospasm with neurotoxin type A: long term results. Eur J Ophthalmol 2003; 13: 331-336. 
2 O'Day J. Use of Botulinum toxin in neuroophthalmology. Curr Opin Ophthalmol 2001; 12(6): 419-422.

3 Steel DH, Hoh HB, Harrad RA, Collins CR. Botulinum toxin for the temporary treatment of involutional lower lid entropion: a clinical and morphological study. Eye 1997; 11(4): $472-475$.

4 Whittaker KW, Matthews BN, Fitt AW, Sandramouli S. The use of Botulinum toxin A in the treatment of functional epiphora. Orbit 2003; 22(3): 193-198.

5 Jabor MA, Kaushik R, Shayani P, Ruiz-Razura A, Smith BK, Morimoto KW et al. Efficacy of reconstituted and stored botulinum toxin type A: an electrophysiologic and visual study in the auricular muscle of the rabbit. Plast Reconstr Surg 2003; 111(7): 2419-2431.

6 Sloop RR, Cole BA, Escutin RO. Reconstituted botulinum toxin type A does not lose potency in humans if it is refrozen or refrigerated for 2 weeks before use. Neurology 1997; 48(1): 249-253.

7 Hexsel DM, De Almeida AT, Rutowitsch M, De Castro IA, Silveira VL, Gobatto DO et al. Multicentre, double-blind study of the efficacy of injections with botulinum toxin type A reconstituted up to six consecutive weeks before application. Dermatol Surg 2003; 29(5): 523-529. 